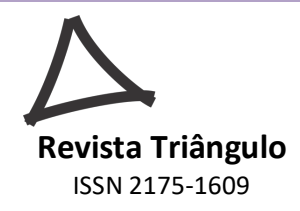

\title{
LAS MUJERES UNIVERSITARIAS DE LA UNIVERSIDAD DE CÓRDOBA Y SUS LUCHAS EN LOS MOVIMIENTOS ESTUDIANTILES
}

\author{
AS MULHERES UNIVERSITÁRIAS DA UNIVERSIDADE DE CÓRDOBA E AS SUAS LUTAS \\ NOS MOVIMENTOS ESTUDANTIS
}
THE UNIVERSITY WOMEN OF THE UNIVERSITY OF CÓRDOBA AND THEIR STRUGGLES IN THE STUDENT MOVEMENTS

María Cristina Vera de Flachs ${ }^{1}$

E-mail: vera@onenet.com.ar

CONICET - Universidad Nacional de Córdoba (UNC)

\begin{abstract}
RESÚMEN
Los estudios históricos en torno al papel que han ejercido las mujeres en la sociedad se han intensificado y quienes se ocuparon del tema, feministas o no, han dado como resultado infinidad de trabajos de investigación con pluralidad de enfoques y diferentes métodos, los que han posibilitado alcanzar conclusiones más ricas y amplias. En numerosas investigaciones se afirmo que nadie se propuso hasta ahora escribir una historia del mundo universitario cordobés de principios del siglo XX desde una perspectiva de género y que quien lo hiciera se encontrará que era un mundo en el que las mujeres estaban ausentes. Opino lo contrario pues, desde el último tercio del siglo XIX, Córdoba tiene ejemplos de mujeres que hicieron oír su voz en ámbitos públicos. En el presente trabajo se analiza la inserción de las primeras mujeres a la Universidad de Córdoba haciendo hincapié en su participación en tiempos de la Reforma de 1918 y años subsiguientes. Ello nos lleva a imaginar el papel potencial de las mujeres de Latino América a lo proceso de integración de la América Latina.
\end{abstract}

PALABRAS-CLAVE: Córdoba. Mujeres. Papel Social y Político.

\section{ABSTRACT}

Historical studies on the role had women in society have intensified and those who dealt with the subject, feminist or not, have resulted in countless research papers with plurality of approaches and different methods, the that you have made it possible to reach conclusions more rich and spacious. In numerous investigations will affirm that no one proposed is now writing a history of Cordoba's early 20 th century academia from a gender perspective and who did find that it was a world in which women were absent. I think the opposite, since the last third of the 19th century, Cordoba has examples of women who made his voice heard in public areas. This paper discusses the inclusion of the first women to the University of Córdoba, with emphasis on their participation in times of the reformation and subsequent years.

KEYWORDS: Córdoba. Women. Social and Political Role.

\footnotetext{
${ }^{1}$ Este trabajo se inscribe también en el proyecto de investigación del grupo HISULA titulado La Universidad Pedagógica y Tecnológica de Colombia en los desafios del siglo XXI. Una prospectiva desde los estudiantes y los docentes. Código SGI 1965 financiado por la UTPC, Colombia 2018.
} 


\section{RESUMO}

Estudos históricos sobre o papel que as mulheres têm desempenhado na sociedade se intensificaram e aqueles que lidaram com a questão, feministas ou não, produziram uma infinidade de trabalhos de pesquisa com uma pluralidade de abordagens e métodos diferentes, que permitiram chegar a conclusões mais ricas e amplas. Em numerosas investigações afirmou-se que ninguém propôs até agora escrever uma história do mundo universitário de princípios córdobos do século XX de uma perspectiva de género e que quem o fez descobriu que era um mundo em que as mulheres estavam ausentes. Eu penso de outra forma, desde o último terço do século $X I X$, Córdoba tem exemplos de mulheres que fizeram suas vozes ouvidas em áreas públicas. No presente trabalho é analisada a inserção das primeiras mulheres na Universidade de Córdoba, enfatizando sua participação nos tempos da Reforma de 1918 e anos subsequentes. Isso nos leva a imaginar o papel potencial das mulheres na América Latina no processo de integração da América Latina.

PALAVRAS-CHAVE: Córdoba. Mulheres. Papel social e político.

Ya que la mujer es la razón del pecado, el alma del demonio, la causa de la expulsión del hombre del paraíso y de la destrucción de la antigua ley, y ya que en consecuencia hay que evitar todo comercio con ella, defendemos y prohibimos expresamente que cualquiera se permita introducir una mujer, cualquiera que ella sea, aunque sea la más honesta en esta universidad.

(Decreto de la Universidad de Bologna, 1377)

\section{Introducción}

Desde hace varios años se vienen publicado infinidad de libros y artículos sobre el papel que han ejercido las mujeres en la sociedad. El feminismo dejó de ser moda y se ha tornado en un reclamo para sacar de la sombra a muchas mujeres que se atrevieron a ingresar en el mundo de los hombres generando reflexión, discusión e información sobre el tema.

Un libro de reciente aparición pertenece a Mary Beard, catedrática de Cambridge que publicó Mujeres y poder. Un manifiesto, donde recorre el lugar que la literatura y la sociedad les otorgaron a las damas a lo largo de los siglos. La autora tiene múltiples publicaciones sobre el tema, lo que le valió que en el año 2016 recibiera el Premio Princesa de Asturias de Ciencias Sociales. Ella sostiene que la voz de la mujer ha sido minimizada a lo largo de la historia incluso por sus características fonéticas. Desde siempre hemos asociado que un buen orador es alguien que logra impostar un tono de voz segura, firme y sobretodo profunda, perdiéndose en esta categorización el contenido del discurso. No obstante debemos señalar que había resistencia de parte del público masculino para reconocer la intromisión femenina en el territorio discursivo de dicho género.

Es decir, los estudios históricos en torno a las mujeres se han intensificado en estos últimos tiempos y quienes se ocuparon del tema, feministas o no, han dado como resultado infinidad de trabajos de investigación con pluralidad de enfoques y diferentes métodos, los 
que han posibilitado alcanzar conclusiones más ricas y amplias. Muchos son los que hacen referencia a los primeros ámbitos donde aquellas desarrollaron sus estudios, trabajos, reclamos y luchas por conseguir derechos similares a los hombres.

Respecto a lo ocurrido en Argentina y particularmente en Córdoba se ha afirmado en numerosas investigaciones que nadie se propuso hasta ahora escribir una historia del mundo universitario cordobés de principios del siglo XX desde una perspectiva de género y que quien lo hiciera se encontrará que era un mundo en el que las mujeres estaban ausentes.

Opino lo contrario pues, desde el último tercio del siglo XIX, Córdoba tiene ejemplos de mujeres que hicieron oír su voz en ámbitos públicos. Tal el caso de la progresista Ignacia Waldina Alba, que en una disertación leída en la Sociedad Unión y Progreso el 31 de julio de 1874, reclamó por la educación de la mujer para evitar estar confinada a un rincón y ser presentada ante la sociedad como torpe y esclava del hombre diciendo entre otros conceptos:

¡Injusta educación! Despojada de su belleza y de sus gracias, despojada de este mérito prestado por pocos años y hallándose desnuda de la verdadera importancia, del valor imperecedero, queda la ciencia...2.

Pero también hubo algunos hombres que sostenían que no había ninguna razón que le impidiese a la mujer sostener "la toga del magistrado, la espada del guerrero o depositar un voto independiente en la urna del sufragio. ¡Educación, educación completa y libre para la mujer y la humanidad marchará unida, sin distinción de clases, de colores, ni de sexos, a la posesión de sus magníficos y celestiales destinos!"3.

Aunque es cierto que desde otro sector algunos autores ejerciendo un paternalismo pensaban lo opuesto y para mostrar sus limitaciones se argüía que las diferencias fisiosicológicas de los sexos incidía en las posibilidades de desarrollo intelectual de la mujer.

$\mathrm{Al}$ acelerarse la transformación socioeconómica algunas mujeres fueron capaces de quebrar los límites de una vida doméstica estrecha y aventurarse en el mundo de la cultura y la educación llegando a aspirar con su ingreso a los estudios superiores.

\section{Los estudiantes y la universidad}

Para comienzos del siglo XX los jóvenes universitarios eran numéricamente pocos en el país y estaban distribuidos de la siguiente manera: la Universidad de Buenos Aires contaba

\footnotetext{
${ }^{2}$ Cfr. Educación de la mujer, Imprenta a cargo de Alberto Ortiz, Córdoba, 1874.

${ }^{3}$ José Miguel OLMEDO, La mujer ciudadana, Córdoba, imprenta del Estado, 1873.

\begin{tabular}{l|l|l|l|l|l} 
(C) Revista Triangulo & Uberaba, Minas Gerais & v.11 & n.4-Esp. & p. $89-108$ & 2018
\end{tabular}
}


con 10.404 alumnos, La Plata con 2.835 y Córdoba con un poco más de mil, mientras las universidades provinciales (Litoral y Tucumán) tenían una matrícula ínfima. Sin embargo, esto no fue un obstáculo para que comenzaran a abandonar su rol pasivo y reclamaran el derecho a ser protagonistas de la vida estudiantil. Así exigieron la actualización de contenidos programáticos, planteando la necesidad de abrir camino a las nuevas corrientes de pensamiento.

La universidad era un polo de atracción para los jóvenes que podían continuar sus estudios en ese nivel, ya que obtener un título profesional aseguraba su ejercicio como medio de vida y alcanzar el grado de doctor -final de la carrera para Derecho y Medicina hasta la Reforma- aseguraba prestigio y facilitaba el acceso al cargo público, tan reconocido socialmente.

Para 1918 la Universidad de Córdoba contaba con 1.001 estudiantes matriculados -es decir menos del 1\% de la población urbana-, incluyendo los locales y los que provenían de otras provincias, principalmente del norte del país. De ese universo, 242 estudiaban en la Facultad de Derecho, 166 en la de Ingeniería y 593, los más numerosos, en la Facultad de Medicina, que tenía dos Escuelas la de Parteras y la de Farmacia. Al igual que lo ocurrido en otras partes del mundo, del total un porcentaje menor eran mujeres, siendo la totalidad en la Escuela de Parteras y menos en la de Farmacia donde las mujeres tuvieron presencia a partir de los primeros años del siglo $\mathrm{XX}^{4}$.

Un hecho que cabe destacar es que la Escuela Normal de Córdoba -como todas las otras que funcionaban en el país-, constituyó en una experiencia exitosa de educación secundaria para las mujeres y preparó el camino para la demanda de estudios universitarios. Sin embargo eso no impidió que ellas afrontaran una serie de polémicas y hasta soportar sospechas en torno a sus capacidades intelectuales y el cómo harían para compatibilizar el ejercicio profesional con los roles de madre y esposa, asignados por el discurso patriarcal. Al respecto, a la mujer se la representaba como débil por naturaleza, dulce, suave y tierna por educación y costumbre, por lo tanto se consideraba que ejercer la más dura de las profesiones como era la de médico era inadmisible.

\footnotetext{
${ }^{4}$ Después de la Reforma el número había crecido en Córdoba a 1.337 estudiantes en total.
} 
Se trataba de las mismas críticas y cuestionamientos que eran dirigidos a otras mujeres que, por entonces, se proponían similares desafíos en Europa e incluso en la Universidad de Buenos Aires. No fue casual que muchas fuesen feministas activas, que militaran por la igualdad de derechos, entre ellos, el acceso a la educación. Y así lo afirmaron varias congresistas que asistieron al Congreso Femenino Internacional que tuvo lugar en Buenos Aires durante 1910, organizado por la Asociación de Universitarias Argentinas con el fin de conmemorar el centenario de la Revolución de Mayo. Entre las asistentes a dicho evento cabe mencionar, entre otras, a Cecilia Grierson, Alicia Moreau, Irma Repetto, Fenia Cherkoff de Repetto, Julieta Lantieri, Leonor y María Teresa Martínez Bisso y varias extranjeras célebres en su época. La reunión cuidadosamente preparada tenía como objetivos establecer lazos de unión entre todas las mujeres del mundo. Vincularlas a un pensamiento común: la educación e instrucción femenina, la evolución de las ideas que fortifiquen su naturaleza, eleven su pensamiento y su voluntad en beneficio de la familia, para mejoramiento de la sociedad y perfección de la raza. Modificar prejuicios, tratando de mejorar la situación social de muchas mujeres.

La doctora Concepción Aleixandre hizo una erudita exposición sobre el papel de la mujer en la medicina a través de la historia señalando cuantas estudiantes de esa carrera había inscriptas en Francia, Bélgica, España, Dinamarca, Suecia y Alemania. Siendo de todos los países europeos Rusia quien tenía más médicas, en 1877 su número era de 430 de las cuales 73 eran judías, 19 católicas y el resto cristianas ortodoxas ${ }^{5}$.

Esto explica que desde el comienzo hubo profesiones que se consideraban "aptas para mujeres", mientras, otras, eran objetiva y subjetivamente masculinas, como abogacía o ingeniería. Cabe preguntarnos entonces ¿cuáles fueron los ideales y anhelos de las precursoras que optaron por las ciencias de la salud en Córdoba?

\section{El caso de Margarita Zaztkin}

\footnotetext{
${ }^{5}$ Argentino J. LANDABURU, Alfredo G. KHON LONCARICA Y Elena PENNINI DE VEGA, "Cecilia Grierson y el primer Congreso femenino Internacional”, en Todo es Historia, número especial, N 183, Buenos Aires, agosto de 1982.
} 
Para explicitar esa pregunta tomaremos el caso de Margarita Zaztkin, quien se animó a ingresar al mundo de los hombres abriendo el camino para que otras mujeres lograran en Córdoba convertir las ciencias de la salud en un sitio donde hoy el género femenino tiene un lugar destacado. Nació el 18 de mayo de 1833 en Odessa - Ucrania. Como tantos otros judíos que huían del antisemitismo, su familia y otros miembros de la colectividad dejaron atrás su tierra mirando con esperanzas a América del Sur. La niña, de escasos 7 años de edad, llegó en el vapor Petrópolis a la Argentina en 1891 junto a sus padres, José y Ana Salman, radicándose en Moisés Ville, colonia establecida en 1889 cuando llegó el primer contingente de migrantes judíos de la mano del rabino Aron Goldman. Ciertamente no sabía leer ni escribir en castellano, por eso en el poblado donde sus progenitores permanecieron por seis años cursó las primeras letras. Sostiene Sandra McGee Deutsch que las mujeres jugaron un papel importante en el proceso de adaptación de los judíos ${ }^{6}$. De sus maestros aprendieron el español y los ideales y costumbres locales, sin embargo contados fueron los casos de niñas judías, que motivadas por sus padres, se atrevieron ir más allá de la instrucción primaria para inscribirse en las escuelas normales que, para fines del siglo XIX, ofrecían prestigio y buen sueldo. Y menos aún las que soñaron con la Universidad.

Debido a que la madre de Margarita padecía enfermedades que eran proclives a curarse en un lugar con buen clima la familia se trasladó a Córdoba, que tenía uno suave y seco, especial para personas delicadas de salud. La vida tranquila, el aire puro y el reposo podían ser una buena práctica curativa. Por otro lado, su padre había conseguido emplearse en una mueblería de esta ciudad, propiedad de un miembro de la colectividad y, a su vez, la familia estaba convencida de la necesidad de lograr una mejor formación para su hija. Estos motivos influyeron para que la familia se instalara en esta ciudad en 1897, incorporándose rápidamente a otro pequeño grupo de inmigrantes hebreos que estaban establecidos desde poco tiempo antes.

La joven decidida a estudiar contó, desde el principio, con el apoyo de su padre, quien le proporcionaba libros para su formación y ello se explica porque la familia procedía de una urbe que, por entonces, tenía casi medio millón de habitantes y donde convivían rusos con armenios, alemanes, tártaros, italianos y judíos con un alto nivel de educación. Ese ambiente tan heterogéneo y multicultural posibilitó en Odessa la existencia de una universidad, gran

\footnotetext{
${ }^{6}$ Sandra MCGEE DEUTSCH “¿Mala fama?” La vida íntima de mujeres judías argentinas, 1890s - 1940s”, University of Texas-El Paso,consultado en línea. estudiosjudios.ides.org.ar/files/2013/07/
} 
cantidad de colegios e institutos y varios periódicos, a la vez que incentivó a que las mujeres ingresaran a la Universidad. Judías, rusas y polacas fueron las primeras que supieron aprovechar la apertura universitaria en Europa, particularmente aquellas que habían sido expulsadas por los pogromos. La mayoría optó por medicina siendo las especialidades más requeridas ginecología y pediatría. Pero, en Córdoba, las cosas eran diferentes y las mujeres tenían escasas oportunidades de formarse e ingresar a la Universidad. Ante esta circunstancia Margarita no se amilanó y si quería inscribirse en la Universidad, previamente debía cursar el secundario en el Colegio Nacional de Monserrat, para lo cual era necesario contar con la aprobación de parte de un tribunal que juzgaría sus conocimientos y la autorización del Ministerio de Instrucción Pública para que fuese admitida en dicho establecimiento educativo. Se entrevistó con el rector de dicho Colegio, entonces un instituto exclusivo para varones, quien al ver su empeño designó una comisión de profesores para que la interrogaran sobre sus conocimientos en literatura, historia y química con el fin que pudiera completar los estudios que le permitieran inscribirse en medicina. Sin embargo, nada le resultaría fácil en tanto su familia sufriría en poco tiempo nuevos traspiés. Falleció su madre en 1902 y poco después su padre quien alcanzó a ver que su hija había concretado su sueño de ingresar a la Escuela de Farmacia, junto a otra descendiente de su colectividad Fanny Bachl Borchardt ${ }^{7}$. Recordar estos casos reviste especial interés porque ilustra el empeño que debieron poner las pioneras para ser aceptadas en la Universidad.

Margarita concluyó la carrera de farmacéutica, con sólo 22 años de edad, en 1905 y tres años, después obtuvo su título de Dr. en medicina y cirugía. Entre sus compañeros figuraban Nicolás Carreras. Miguel A. Ferrer, Carlos Fábrega Luque, Pedro Cometo Senestrari, Manuel Masjoan, Bernardo Lozada Echenique, Javier Humberto Lascano Colodrero, Efraín Martínez Zuviría, León Morra y Manuel Ninci, muchos de ellos con sobresaliente actividad en la Universidad de Córdoba en los años subsiguientes.

Tras aprobar las materias del séptimo año con notas sobresalientes Margarita comenzó a pensar en el tema para redactar su tesis doctoral decidiéndose por uno relacionado con su sexo. Así se abocó a analizar el caso de una parturienta con distocia que ella había tratado cuando estuvo junto con su compañero Manuel Ninci, en la sala de ginecología del Hospital San Roque, entonces a cargo de los doctores Gil Barros y Benjamín Galíndez. Luego redactó de su puño y letra la tesis doctoral titulada "Un caso de distocia por un quiste hidatídico" que

\footnotetext{
${ }^{7}$ Fanny Bachl, se había recibido de partera en 1901.
} 
dedicó a la memoria de sus padres. Fue su director el joven Dr. Elías Fernández de la Puente, miembro de una familia liberal criolla de Entre Ríos y ex alumno de la Universidad de Córdoba, con quien casó en 1910 y tuvo tres hijos ${ }^{8}$. Ella casi no ejerció la profesión pues falleció a los 44 años en la ciudad de Rosario?.

A partir de estos primeros casos, poco a poco la mujer fue incorporándose a las aulas universitarias y posibilitó que recibiera igual formación que los varones. Después de la Primera Guerra Mundial las argentinas de clase media incrementaron su presencia en ella, aunque de manera desigual respecto al hombre, siendo preferida por las jóvenes la carrera de farmacéutica que demandaban menos tiempo de estudio.

A Margarita Zaztkin le siguieron: en 1917, la santafesina Amparo Lafarga que obtuvo el mismo título a los 24 años de edad y otras que rindieron en 1921 reválidas como la española Isabel Rodríguez y la rusa Fruna Wolobrinsky de Erugimovich. Ese mismo año se recibieron como doctoras en odontología las argentinas Ana María Coromidas y María Del Valle Giménez y como farmacéuticas las argentinas Felipa Antonia Ávila, Amelia R. Bruno, y Lucía Pagano ${ }^{10}$.

Hoy más del 50\% en varias carreras son mujeres. Es decir en el ámbito universitario a lo largo de un siglo la mujer ha hecho una revolución histórica, sin embargo los cambios en algunos estamentos de poder todavía no tienen la rapidez que se necesita.

\section{La mujer en el mundo reformista}

Si bien es cierto que el mundo reformista era mayoritariamente masculino, hubo mujeres que se animaron a romper con los prejuicios e ir más allá, acompañando a los compañeros del otro sexo.

La Escuela de Parteras ofrecía un espacio de formación superior y reconocimiento práctico, así como la posibilidad de revalidar los títulos adquiridos en otras universidades. Para ingresar a la Escuela había que tener como mínimo 20 años, haber rendido examen con aprobación hasta el sexto grado, "tener buena conducta, gozar de buena salud, carecer de

\footnotetext{
8 Sus hijos fueron: Susana Raquel (1911-2006); Edgard Elías (1913-1974) y Elías Federico Aníbal (1917 1998?).

9 María Cristina VERA DE FLACHS, «Las primeras mujeres universitarias en Córdoba (Argentina) y la Escuela de Parteras». Revista de Historia de la Educación Latinoamericana, No. 3, Santa Fe de Bogotá, Colombia, 2001.

${ }^{10}$ Archivo General e Histórico de la UNC www.archivodelauniversidad.unc.edu.ar Índice de las primeras mujeres egresadas de la Universidad Nacional de Córdoba. 1884 - 1950.

\begin{tabular}{l|l|l|l|l|l} 
(c) Revista Triangulo & Uberaba, Minas Gerais & v.11 & n.4-Esp. & p. $89-108$ & 2018
\end{tabular}
}


impedimento intelectual y no tener imposibilidad física para el ejercicio de la profesión"11. En este punto, cabe mencionar que el requisito de no tener "impedimento intelectual", no aparece en la reglamentación de lo solicitado para cursar la carrera de doctorado en medicina ni para la de farmacia; carreras que cuando se formalizaron no se contempló expresamente que pudieran ser cursadas por mujeres. Sin embargo, a los pocos años, se graduaron las primeras médicas y farmacéuticas. En este sentido, las estudiantes de la Universidad Nacional de Córdoba no fueron una excepción. En casi todos los países europeos y latinoamericanos, las primeras universitarias fueron egresadas de las Facultades de Medicina porque el impulso a la medicina parecía ser natural en las mujeres, tan natural como la enseñanza, pues las esposas y madres eran a comienzos del siglo XX como lo habían sido siempre las supervisoras de la salud y las enfermeras en el hogar.

\section{Nace la Asociación Córdoba Libre}

Un grupo de jóvenes que habían cursado sus estudios de leyes en la Universidad de Córdoba decidieron aunar esfuerzos para formar, en 1916, la Asociación Córdoba Libre. Su origen tuvo lugar en una reunión en el Hotel Plaza en la que participaron entre otros líderes reformistas Deodoro Roca, Saúl Taborda, Arturo Orgaz y Arturo Capdevila, quienes resolvieron emprender la construcción de un espacio democrático y plural, lo que dio lugar para que el 10 de setiembre de 1916 se constituyera la "Asociación Córdoba Libre”, presidida por Arturo Orgaz. Adhirieron a ella además de los nombrados, Amado J. Roldán, Julio H. Brandán, Rafael Bonet, Martín Gil, Luis León, Octavio y José Pinto (hijo), Félix Etchegaray y José y Benjamín Palacio. La asociación tuvo carácter contestatario y exhibió una nueva sensibilidad que fue reflejo de la actividad intelectual, política y social de esa generación que dio como resultado la reforma de $1918^{12}$.

Córdoba Libre incluyó un Comité Femenino, representado por María Ofelia Grandoli y Ana Mori que, a su vez, eran delegadas de la Escuela Profesional de Señoritas y ejercieron ese papel en 1918, acompañando a los compañeros reformistas. Es decir desde diferentes ámbitos las mujeres se fueron sumando a la lucha. Esta aseveración lo prueba también un

11 M. C. VERA DE FLACHS, De comadronas a obstetras: la institucionalización del saber - 1a ed. - Báez Ediciones, Córdoba, 2010.

${ }^{12}$ M. C. VERA DE FLACHS y Jorge GAITERI "La Asociación Córdoba Libre a la luz de nueva documentación” en Hacia los cien años de la Reforma de 1918, Córdoba, 2018.

\begin{tabular}{l|l|l|l|l|l} 
(C) Revista Triangulo & Uberaba, Minas Gerais & v.11 & n.4-Esp. & p. $89-108$ & 2018
\end{tabular}


artículo cuyo título es La rueca de Boleslao que apareció en la Revista La Montaña, órgano oficial de esa agrupación, donde se expresa lo siguiente:

La manifestación pública del domingo ha renovado el recuerdo de aquel episodio. Ha sido necesario que las mujeres abandonaran sus faenas: sus hogares, sus hijos, sus cocinas y las tiendas para que la causa del "orden establecido" de la "fe de nuestros mayores" y de las instituciones eclesiásticas tan caras a Episcopos y Cía. tuvieran una "entusiasta" defensa que no han sabido prestarle los Boleslao de Córdoba.

Evidentemente aparece aquí una clara referencia a la presencia de las mujeres cordobesas y su participación activa en la vida pública al punto que se señala que "ellas salen a las calles, pronuncian arengas, dicen cosas feas y hacen cosas de cabarets"13.

Sin embargo en algunos sectores católicos las arengas y los cánticos de los muchachos reformistas que iban contra la Iglesia y la fe como aquel que decía "corazón santo tu reinarás pero los frailes nunca jamás pasarán” o "con la tripa del último fraile se hará la horca para el último rey" cantados en las manifestaciones callejeras y escritos por Arturo Capdevila, asustaban a las beatas madrugadoras y revolucionaban a las familias católicas despertando miedo. Por eso cerraban sus puertas y ventanas arguyendo que era probable que estos jóvenes hasta quisieran violarlas. Ese era el argumento que utilizaban a la noche al cerrar las puertas las monjas del Colegio de las Hermanas adoratrices a las estudiantes que estaban internas, según recuerda una hija de éstas ${ }^{14}$.

Otra muestra de la incipiente participación de la mujer en la vida pública y la admiración que despertaba Deodoro Roca en el universo femenino, es la tarjeta encontrada en los papeles privados de éste donde alguien que se manifiesta parienta y amiga y dice lo siguiente:

Tarjeta:

Señor

Dr. Deodoro Roca.

Con entusiasmo te

Saludo felicitándote por

el valor y la franqueza

deseando el más bril-

lante éxito á la idea

\footnotetext{
${ }^{13}$ La Montaña, publicación de Córdoba Libre, año I Num I. Córdoba, 24 de agosto de 1918, pag.7.

${ }^{14}$ Entrevista realizada a la hija de un reformista este año.YVdeL.
} 


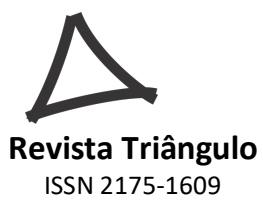

de ¡Córdoba libre!

Afectos a las muchachas

Y un efusivo apretón de

Manos para ti de tu

parienta y amiga

Josefina Torres Cabrera ${ }^{15}$

Y porque no mencionar los celos que sentía su entonces novia Maruca Deheza que le decía a Deodoro que tuviera cuidado con la Señorita Morini que era una activa reformista y aparentemente se movía en ese círculo.

\section{La lucha estudiantil}

En los primeros años del siglo XX encontramos en la Universidad de Córdoba graduadas relacionadas con el mundo de la medicina: parteras, farmacéuticas, odontólogas y doctoras en medicina y cirugía. No fue fácil para estas jóvenes cursar las materias en medicina y en odontología pues no se les permitía compartir las mesas examinadoras y los trabajos prácticos con sus compañeros varones, que les gastaban bromas pesadas con ciertas partes íntimas de los cadáveres, las que solían colocar en los bolsillos de sus guardapolvos. También el matrimonio y la crianza de los hijos produjo que, a veces, la obtención del título se demorara y no se recibieran a término, tal el caso de la santafecina Prosperina Justa Parován que se casó con un compañero de estudios y de lucha -Marcelo Germain- y con quien compartió la presidencia y vicepresidencia de la Comisión Directiva del Centro de Estudiantes de la Escuela de Odontología de los años 1918-191916.

Prosperina consiguió que su padre la autorizara a viajar a Córdoba para inscribirse en la carrera de Odontología. Y su adaptación a esta ciudad fue tal que, en 1918, estaba inmersa en la lucha estudiantil. Muchas otras también transitaron los increíbles días de la Reforma de 1918, pero su participación resulta hasta hoy imperceptible aun cuando hay ejemplos concretos de su intervención.

En junio de ese año los miembros del Comité Pro Reforma Universitaria, querían la creación de una Federación de Centros de Estudiantes como la que se produjo por esos días a nivel nacional con el surgimiento de la Federación Universitaria Argentina, por la que

15 Bibliorato II Documento TUV 42-, papeles privados.

${ }^{16}$ Se recibió el 14 de abril de 1936 con el grado de Odontóloga. 
propiciaron elecciones en todos las Facultades. En Ciencias Médicas lo eligieron a Enrique Barros, que estudiaba medicina y eso provocó cierto recelo en las Escuelas que dependían de esa Facultad, las que tardíamente fundaron el Centro de Estudiantes de Farmacia y el Centro de Estudiantes de Odontología.

En una reunión en el Centro de Estudiantes de Ciencias Médicas y bajo la presidencia de Prosperina aceptaron la invitación de la FUC para enviar a su seno un delegado provisorio por Odontología firmando ella e Inés Rosenblatt junto a otros compañeros la nota correspondiente donde decidieron que no adherirían a la Federación y mantuvieron una posición crítica con los reformistas.

Prosperina no coincidía con esas decisiones arbitrarias y de celos varoniles por el protagonismo político, más aun cuando producidos los hechos del 15 de junio, su Centro de Estudiantes había sido rechazado y desconocido en la FUC, por lo que eligió una posición crítica para con ambos bandos en pugna. No obstante ella igualmente marchó por las calles y enfrentó la represión de la policía que poco caso le hacía al gobierno de Borda. En esa ocasión pudo conversar con Enrique Barros, que auspició el acto de revelarse a la moral conservadora de reconocer a la mujer y acordaron convocar a la asamblea de estudiantes de Odontología para determinar si adherirían o no a la Reforma y a la FUC. Así fue que el 19 de junio se reunieron los estudiantes de Odontología, en el Centro de Estudiantes de Ciencias Médicas y bajo la presidencia de Prosperina, resolvieron: $1^{\circ}$.- Aceptar con viril entusiasmo la invitación de la Federación Universitaria de Córdoba, para enviar a su seno, un delegado provisorio. $2^{\circ}$.Nombrar al señor Vicente Decoud para llenar ese cometido. $3^{\circ}$.- Munirlo del presente documento firmado por todos los adheridos, que lo acredite en su carácter de delegado.

Firmado: por dos mujeres Prosperina Paraván e Inés Rosenblatt y por Santiago Palacios, R. Santillán, Alberto R. Vidal, Elías Discowsky, J.A. Balzaretti, Roberto Libedinsky, F. Lanza Castelli, A. Barbalat, J.M. Lurá, Luís Sigal, V. Decoud, Martín Torres, Gregorio Dicowsky, José Luís Silva (h) ${ }^{17}$.

\section{El ingreso de las mujeres en otras carreras universitarias}

La década del 20 trajo profundas transformaciones para la ciudad de Córdoba y su Universidad que atravesaba los gloriosos cambios de la Reforma incluida la presencia de la

\footnotetext{
${ }^{17}$ La mujer que construyó la Reforma Universitaria, Cf. Claudio BUSTOS, martes, 6 de febrero de 2018, en línea.
} 
primera notaria y la segunda en el país: Mercedes Orgaz, que provenía de una típica familia cordobesa conformada por una madre, doña Mercedes Ahumada que había solo cursado hasta tercer grado en su pueblo natal aunque de una gran inteligencia para educar a sus hijos que estaban dejando huellas en sus respectivas carreras y un padre Don Eleodoro Orgaz de fuerte carácter pero con profundas inquietudes sociales ${ }^{18}$.

La reforma universitaria sorprende a Mercedes en el ámbito doméstico aunque informada por sus hermanos de lo que estaba ocurriendo. En esos momentos contaba con 24 años y soñaba con ingresar a medicina donde su hermano Jorge estaba cursando sus estudios. Pero a pesar del progresismo de sus hermanos no encontró eco, pues ellos pensaban que era una carrera muy compleja para una mujer.

De todos modos no se acobardó y decidida a estudiar una carrera universitaria, el 22 de octubre de 1919, en nota al rector del Colegio Nacional de Monserrat, doctor Fidel Bazán, solicitó permiso para rendir libre el primer año en dicho instituto, fijando domicilio en 27 de Abril 890 y aprobando todas las asignaturas. El segundo año lo cursó como alumna regular en el Liceo Cultural, un instituto privado incorporado al Monserrat, nacido de un proyecto del padre Luis Feliú -sacerdote jesuita con cuya obra social simpatizara Mercedes- para facilitar la educación de la mujer. Allí convergió, como dice Alfredo Rosetti, "una generación pionera de jóvenes profesionales que debió completar su bachillerato en el Colegio Nacional de Monserrat, como Zoé Bialet Laprida (escribana), Angelina Amuchástegui (bioquímica), Lola Moyano López (bioquímica), Zulema Bialet Laprida (médica) y Elisa Ferreyra Videla (abogada)"19.

En noviembre de 1920 Mercedes finalizó con todas las materias de segundo año, excepto Historia, que rendirá luego en el Monserrat donde entre diciembre de 1920 y julio de 1921, aprobará como alumna libre todas las asignaturas de tercer año.

En noviembre de 1921, Mercedes completa el cuarto año y en diciembre de 1921, sólo un mes después, aprueba como libre todas las materias de quinto año. A su solicitud, le es expedido el certificado analítico, que acredita su condición de bachiller, el 21 de julio de

\footnotetext{
${ }^{18}$ Carlos A. IGHINA, Mercedes Orgaz, primera notaria universitaria de Córdoba, consultada en línea.
}

${ }^{19}$ IDEM, Ibídem. Alfredo C. ROSETTI, Universitarios de Córdoba. Córdoba 1980, Biffignandi Ediciones. pág. 40 . 
1922, con la firma del secretario, M.A. Escalera, y del rector, ingeniero Rafael Bonet. En dos años y dos meses había terminado sus estudios secundarios.

El 7 de agosto de 1922, Mercedes solicitó matrícula para cursar estudios en la Escuela de Notariado, sentando con ello un importante precedente, pues pasaba a ser una de las primeras mujeres ingresantes a la Facultad de Derecho y Ciencias Sociales. Luego de poco más de 16 meses de estudio, sobre un plan de tres años, Mercedes recibió el título de notaria el 13 de diciembre de 1923, ante la presencia orgullosa de sus hermanos. El rector de la Universidad, doctor Ernesto Romagosa, le hizo entrega del diploma que la acreditaba, además, como la primera egresada de la mencionada Facultad de Derecho y Ciencias Sociales, lo que le posibilitó ejercer su profesión con idoneidad y gran pericia. Contemporáneas suyas fueron Zoé Bialet Laprida también notaria, y Elisa Ferreyra Videla, la primera abogada que egresaría con ese título el 23 de agosto de 1929 y el 20 de setiembre de 1938 con el grado de Doctora en derecho con una tesis titulada Hacia una organización corporativa. En 1941 Elisa se incorporará como profesora adjunta en la cátedra de Economía Política y como titular en $1947^{20}$.

Sin lugar a dudas, el ingreso a estas carreras liberales fue un poco más tardío respecto a la de medicina y sus respectivas escuelas, ya que estaban ligadas con el prestigio y el poder. Ellas debieron compartir las aulas con sus compañeros, repitiendo en las clases de derecho civil, su singular consideración de incapaces relativas de hecho y en derecho constitucional por la falta de derechos políticos.

\footnotetext{
${ }^{20}$ Falleció en Córdoba el 21 de noviembre de 1979. Ramón Pedro YANZI FERREIRA "Elisa Ferreyra Videla. Primera graduada de la Facultad de Derecho y los estudios de Economía Política en la Universidad Nacional de Córdoba. 1947-2010" en Cuadernos de Historia, Nro 20, editorial Advocatus, Córdoba, 2011, pp. 27 a 58.
} 


\section{La lucha continua}

En los años treinta y después del primer golpe de Estado la violencia en la Universidad no se detenía. Conservadores y la derecha nacionalista se enfrentaban a diario con los reformistas.

En marzo de 1932, siendo presidente de la Nación el Gral. Justo los estudiantes reformistas, agrupados en la Federación Universitaria se declararon en huelga ante el avance de los sectores reaccionarios y por la exoneración de varios profesores de Medicina: doctores Jorge Orgaz, Gumersindo Sayago y Tomás de Villafañe Lastra tres estudiantes reformistas de 1918 y Gregorio Bermann. Y en Derecho el Dr. Donato Latella Frías de reconocida adhesión al radicalismo. Se sumaron a ello detenciones de docentes y de 141 estudiantes, lo que fueron defendidos por varios abogados reformistas como Deodoro Roca, Alfredo Orgaz, Marcelo Posse, J. Ahumada y Salomón Roitman. Hubo enfrentamientos físicos en el ámbito universitario y en el Colegio Nacional de Monserrat donde se agredió a dos estudiantes que repartían la revista Estudiantina.

En esa lucha que alcanzó proyecciones extraordinarias y largos meses de huelga descolló el joven estudiante de medicina Tomás Bordones y su compañera Leticia Aguirre que estuvo al pie de la misma junto a otras mujeres ${ }^{21}$. Por su parte las alumnas del Liceo de Señoritas adhirieron a la huelga y manifestaron su adhesión y solidaridad con los huelguistas. Ello explica que en el acto realizado el 16 de junio de 1932 en el teatro Rivera Indarte para conmemorar el décimo cuarto aniversario de la Reforma numerosas damas se ubicaran en los palcos ${ }^{22}$. Sin embargo ninguna de ellas figuran en las listas de las elecciones estudiantiles.

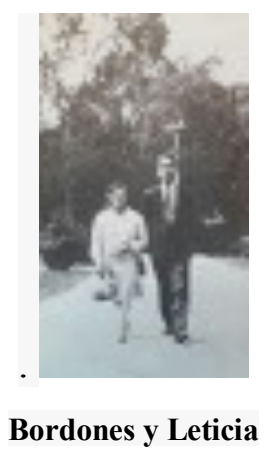

${ }^{21}$ Leticia a pesar de estudiar en esos años no se recibió de médica. Para quien quiera profundizar este tema Leticia AGUIRRE, La generación del 32. Reforma universitaria. Tomas Bordones, prólogo de Raúl Faure, Editorial Universidad de Córdoba, 1989.

${ }^{22}$ La Voz del Interior, 17 de junio de 1932. 
A pesar de esa dilatada huelga nada cambió en la Universidad. El 15 de junio de 1936 en el Teatro Rivera Indarte, en un nuevo acto por conmemorar la reforma los oradores del grupo coincidieron en denunciar que la institución estaba en manos de fascistas y que los problemas del estudiantado estaban "en el mismo lugar que en 1918"23. Cabe destacar que los actos públicos de la Federación Universitaria de Córdoba (FUC) -prohibidos por el rectoradocontaban en esta época con la presencia de un entramado de asociaciones que se nutrían de los impulsos de los intelectuales reformistas, incluyendo la novedosa organización de mujeres pacifistas titulada Unión Femenina Anti guerrera (AMA) a semejanza de otras asociaciones conformadas en España y otros lugares de América.

\section{A modo de cierre}

Hemos tratado de reseñar en estas páginas la inserción de la mujer en la Universidad de Córdoba desde las últimas décadas del siglo XIX, haciendo hincapié en lo sucedido en los años de la reforma de 1918 y posteriores cuando ellas empiezan a participar tímidamente de las luchas estudiantiles y cuando se graduaron en otras profesiones liberales fuera de las dependientes de la Facultad de Medicina como la de notaria y abogacía y aquellas consideradas como "masculinizadas" como la arquitectura y la ingeniería civil.

En los años cuarenta aparecieron las primeras peritos traductoras y profesoras de francés, inglés, alemán e italiano y la graduación de las primeras doctoras y licenciadas en Filosofía; a las que se sumaron, contadoras públicas, geólogas y doctoras en ciencias naturales.

En los años sesenta gran cantidad de mujeres ingresaron a distintas carreras universitarias en Argentina y en la Universidad de Córdoba en particular. Los problemas políticos de esos años y de la década siguiente las encuentran participando de los centros estudiantiles, e inmersas en la lucha dentro y fuera de los claustros igualando casi en número a los varones. Al igual de lo ocurrido en otros puntos del planeta, era una revolución cultural contra los gobiernos autoritarios, el clero y la familia. El Cordobazo y la lucha contra el

\footnotetext{
${ }^{23}$ Gaceta Universitaria, órgano de la FUC, 21 de mayo de 1932, Num. 4, Córdoba.
} 

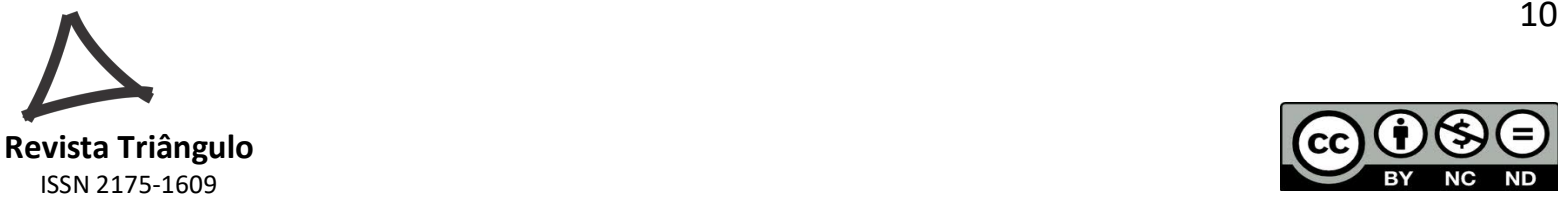

gobierno del general Juan Carlos Onganía y luego contra el gobierno del general Rafael Videla las encuentran militando activamente.

La mayoría de las mujeres que luchaban en esa etapa se enrolaron en el Partido Comunista y en la JTP, dependiente del partido justicialista y en los distintos grupos guerrilleros de la época como Las Fuerzas Armadas Revolucionarias (FAR), las Fuerzas Armadas Peronistas (FAP), las Fuerzas Armadas de Liberación (FAL), el Partido revolucionario de los Trabajadores (PTR), el Ejército Revolucionario del Pueblo (ERP) y Montoneros $^{24}$.

Durante los años setenta la subversión mantuvo e intensificó sus ataques que fueron reprimidos por las fuerzas armadas. A la violencia se respondió con violencia, lo que impedía el normal funcionamiento del país, al tiempo que el cuerpo social en general estaba atemorizado ante lo que se reflejaba en la opinión pública como una guerra abierta entre grupos facciosos y el ejército.

En síntesis, la lucha por conquistar un lugar en la UNC y en la sociedad en general fue dura a lo largo del siglo posterior a la Reforma, pero hoy las mujeres son mayoría en el sector estudiantil, en el profesorado y entre las no docentes. Sin embargo el techo de cristal continúa para obtener los cargos jerárquicos ${ }^{25}$.

\footnotetext{
${ }^{24}$ En Córdoba destacaron, entre otras, Marta Sagadin, ex militante del Partido Comunista, fundadora de la Bibloteca de la mujer Juana Manuela Gorriti; Cristina Salvarezza, estudiante de arquitectura y militante del PRT que participó en la fuga de mujeres de la cárcel del Buen Pastor en 1975, Ana María Peña bancaria y militante de la JTP, Graciela Ferreyra Campos, estudiante de Derecho y militante de izquierda.Muchas perdieron su vida y, otras, estuvieron presas en condiciones infrahumanas.

${ }^{25}$ Desde 1986 en adelante se realizan encuentros nacionales de mujeres en distintas ciudades del país con el objetivo de buscar la transformación social. Es decir, en este espacio ellas encuentran una herramienta para aprender, retroalimentarse, empoderarse y articularse mutuamente, para luego trasladar toda esta práctica y conocimiento a sus respectivas comunidades. Esos encuentros se caracterizan por ser autónomos, autoconvocados, democráticos, pluralistas, autofinanciados, federales y horizontales.
} 

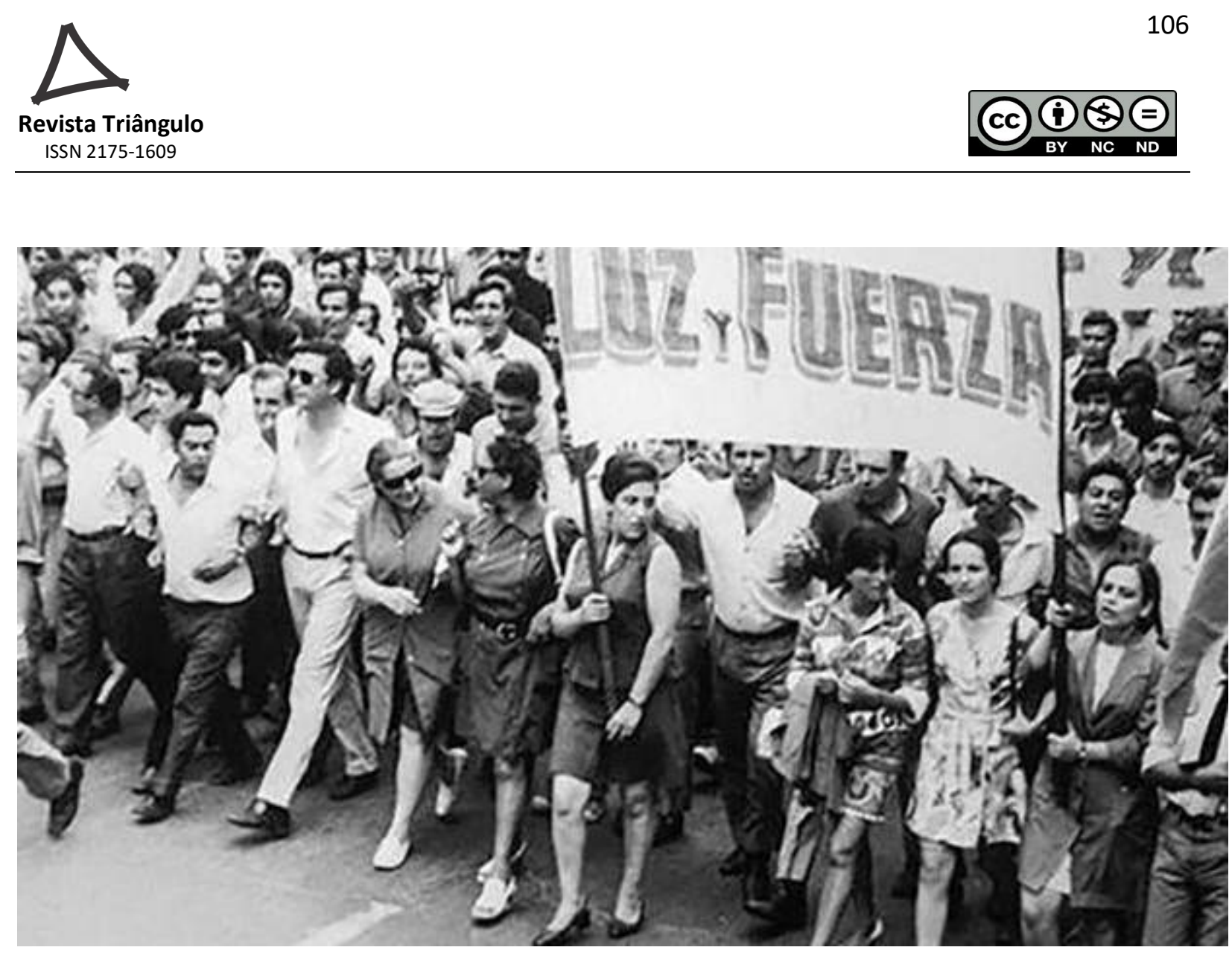

Foto Bibiana Fulchieri

\section{FUENTES}

\section{La Gaceta Universitaria}

La Montaña, publicación de Córdoba Libre, año I Núm. I. Córdoba, 24 de agosto de 1918, pag.7.

La Voz del Interior

Bibliorato II, Documento TUV 42-, papeles privados.

ARCHIVO DE CIENCIAS DE LA SALUD, A.C.S, Tesis Originales, 1 era Serie.

\section{REFERÊNCIAS}

AGUIRRE, L. La generación del 32. Reforma universitaria. Tomas Bordones, prólogo de Raúl Faure, Editorial Universidad de Córdoba, 1989.

ALBA, I. W. Educación de la mujer, Imprenta a cargo de Alberto Ortiz, Córdoba, 1874.

BEARD, M. Mujeres y poder. Un manifiesto. Editorial Planeta: España, 2018. 
BLANK, B. La mujer judía en la ciudad de Córdoba a comienzos del siglo XX. Junta Provincial de Historia de Córdoba. Córdoba, 1994.

BUSTOS, C., La Mujer que construyó la Reforma Universitaria. Martes, 6 de febrero de 2018. www.reformadel18.unc.edu.ar/.

CORTES, N.; FREYTES, A. Índice de las primeras mujeres egresadas en la Universidad Nacional de Córdoba 1884-1950 / Nuria Cortes. - 1a ed. - Córdoba: Editorial de la UNC, 2015. Libro digital, PDF Archivo Digital: descarga y online.

IGHINA, C. A. Mercedes Orgaz, primera notaria universitaria de Córdoba. Consultado en línea: http://escribanos.org.ar/rnotarial/wp-content/uploads/2015/07/RNCba-71-1966-04Doctrina.pdf

LANDABURU, A. J.; G. KHON LONCARICA, A.; PENNINI DE VEGA, E. Cecilia Grierson y el primer Congreso femenino Internacional. En Todo es Historia, número especial, N 183, Buenos Aires, agosto de 1982.

MCGEE DEUTSCH, S. ¿Mala fama?” La vida íntima de mujeres judías argentinas, 1890s 1940s. University of Texas-E1 Paso, consultado en línea: http://ides.org.ar/archivos/6181 estudiosjudios.ides.org.ar/files/2013/07/

OLMEDO, J. M. La mujer ciudadana. Córdoba: imprenta del Estado, 1873.

ROSETTI, A. C. Universitarios de Córdoba. Córdoba: Biffignandi Ediciones, 1980.

VERA DE FLACHS, M. C. Darwinismo, Naturalismo y Mujer. En Primer Congreso binacional Argentino- Chileno, Minorías y marginalidad en Estados Unidos y Latinoamérica. Valdivia, Chile: Edic. del Instituto Chileno Norteamericano de Cultura, 1992.

VERA DE FLACHS, M. C. Mujeres en los claustros universitarios. La Escuela de Parteras. 1884-1970. En III Jornadas de Historia de Córdoba. Junta Provincial de Historia de Córdoba, 1997.

VERA DE FLACHS, M. C. Mujeres Universitarias. El caso de Córdoba. En SMITH, Y. D. y GONZÁLEZ, M. C. Mujeres en el mundo: Género, trabajo, salud, educación, arte, cultura y redes en movimiento.(Coord). Universidad de Carabobo, Valencia, Venezuela, Junio de 2006.

VERA DE FLACHS, María Cristina, «Las primeras mujeres universitarias en Córdoba (Argentina) y la Escuela de Parteras». Revista de Historia de la Educación Latinoamericana, No. 3, Santa Fe de Bogotá, Colombia, 2001. 
VERA DE FLACHS, M. C. Reformas y contrarreformas y movimientos estudiantiles en la Universidad de Córdoba, 1870-1936. En MARSISKE, R. (Coord.) Movimientos estudiantiles en la historia de América Latina, III. Universidad Nacional Autónoma de México, México, 2006, pp. $21-81$.

VERA DE FLACHS, M. C. De comadronas a obstetras: la institucionalización del saber. 1a ed. - Córdoba: Báez Ediciones, 2010.

VERA DE FLACHS, M. C.; GAITERI, J.. La Asociación Córdoba Libre a la luz de nueva documentación. En Hacia los cien años de la Reforma de 1918. Córdoba: Baez Editora, 2018.

YANZI FERREIRA, R. P.; Elisa Ferreyra Videla, primera graduada de la Facultad de Derecho y los estudios de Economía Política en la Universidad Nacional de Córdoba. 19472010. En Cuadernos de Historia Nro. 20, Córdoba: Editorial Advocatus, 2011, pp. 27-58. 